\title{
Experimental and dynamic study of the piston rod lateral friction for the twin-tube hydraulic shock absorber
}

\author{
Yanqing Liu, Jianwu Zhang* and Xiaoming Cheng \\ Institute for Automotive Engineering, School of Mechanical Engineering, Shanghai Jiaotong University, Shanghai, \\ 200030, P.R. China
}

Received 31 July 2001

Revised 24 September 2002

\begin{abstract}
In this paper, dynamic loads acting on a twin-tube hydraulic shock absorber are derived out both in wheel and axle planes by modeling mechanically car rear suspensions, and internal and external forces that yield lateral surface damage and wear-out of the piston rod for the absorber are analyzed according to bench and real road test measures. From viewpoint of vehicle system dynamics and experiment, such key factors as road unevenness, very high car speed and severe shock induced vibrations are investigated, by which stochastic bending moments and dramatically increasing shock loading are introduced directly to the piston rod. From viewpoint of the whole car assembly, on the other hand, due to hardly perfectly placements of the piston rods in their positions between the car suspension and body, unacceptable manufacturing quality of the body may cause additional dynamic forces on the piston rod. Significant results obtained by theoretical and experimental analysis of lateral frictions of the piston rod are presented systematically for improving design of the shock absorber.
\end{abstract}

Keywords: Experimental mechanics, car suspension, shock absorber, signal treatment

\section{Introduction}

The twin-tube hydraulic shock absorber is one of important components in car suspension, which can attenuate vibration and enhance car compliance and comfort by transforming kinetic energy of vibration between the car wheel and body into heat energy through certain orifices. With increase of car driving speed, the shock absorbers have many practical problems caused by the lateral friction between the piston rod and the seal, which may lead to function loses in an early stage of such use as piston rod wear-out and oil leakage [13]. Some research workers have made much effort in the development of dynamic modelings and bench test results for the absorbers [2-4]. In spite of this, there

\footnotetext{
${ }^{*}$ Corresponding author: Tel. and Fax: +8621 6293 3772; E-mail: jwuzhang@mail.sjtu.edu.cn.
}

is still lack of real car road experiments and dynamic modeling information for lateral frictions of the piston rods. In the present research, great effort has been made to undertake not only bench tests but also real car experiments to get practical information for dynamic modeling and statistic analysis of mechanical tribological behaviors of the absorbers.

\section{Experiment and dynamic modeling}

The rear suspensions with the twin-tube hydraulic shock absorbers are given in Fig. 1 and the motion mechanism of a rear suspension in the wheel plane is shown in Fig. 2, in which spherical joints between the body and shock absorbers are defined in the suspension systems. The plane joint of revolution between the absorber and the rear trailing arm is so defined that 


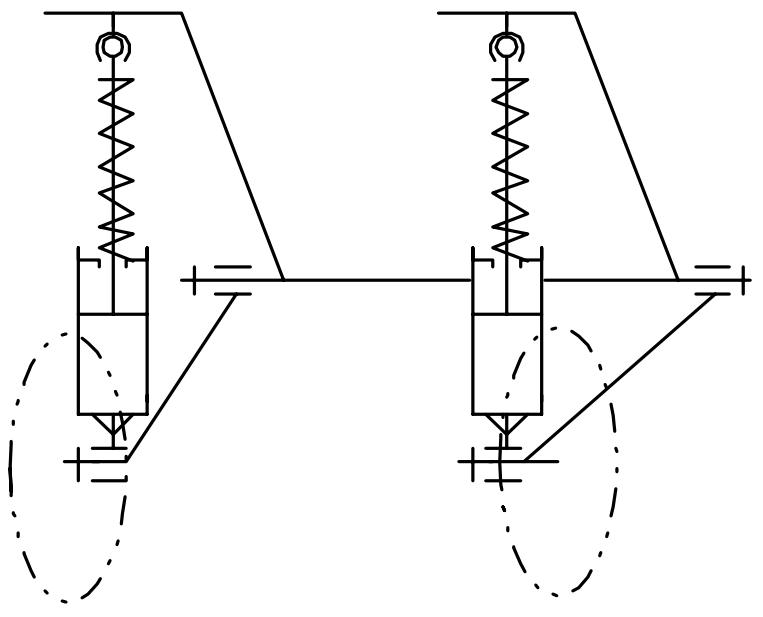

Fig. 1. The rear suspensions system with shock absorbers.

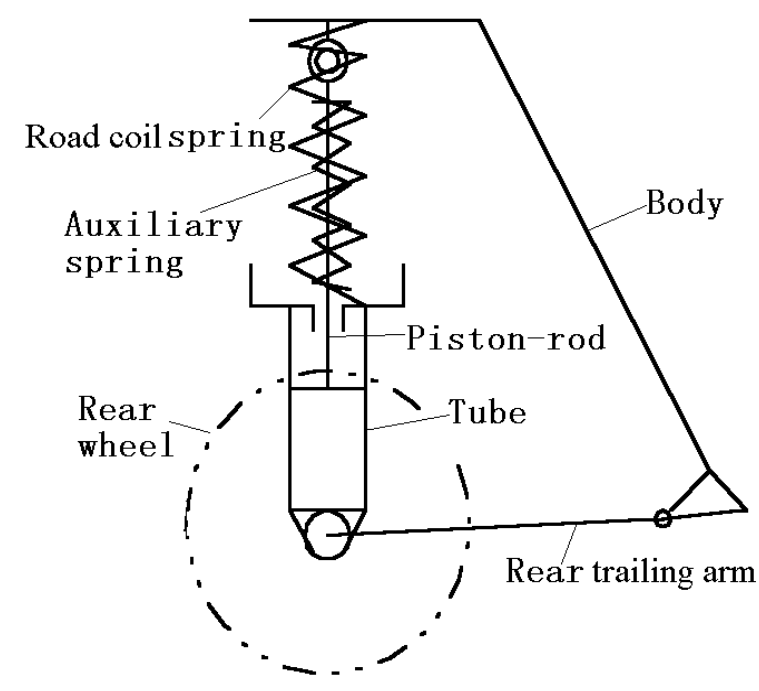

Fig. 2. The motion mechanism of a rear suspension in the wheel plane.

the shock absorber is rotatable in the wheel plane, but restrained in the axle plane. In Figs 3 and 4 are presented the dynamic models for the rear suspensions and twin-tube hydraulic shock absorber.

In Fig. 4, $O$ and $O^{\prime}$ represent the spherical joints between the absorber piston rod and the body and the hinge joint between the absorber tube and the rear trailing arm. $F_{1}, F_{2}, F_{12}$ and $F_{13}$ are reaction forces acting on the tube by the rear trailing arm at Point $O^{\prime}, F_{6}, F_{7}$, $F_{10}$ and $F_{11}$ also reaction forces on the piston rod by the body at Point $O, M_{\varphi 1}$ and $M_{\varphi 2}$ moments applied by the body to the piston rod, and $M_{B}$ a moment applied due to the tube clamped boundary condition in the axle plane, respectively.

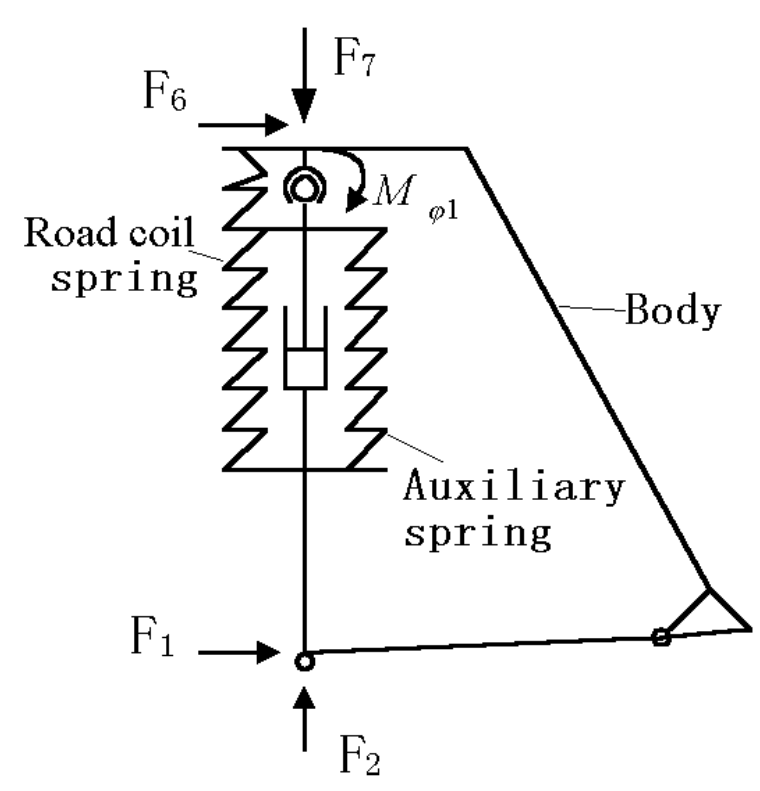

Fig. 3. Dynamic model of the rear suspension in the wheel plane.

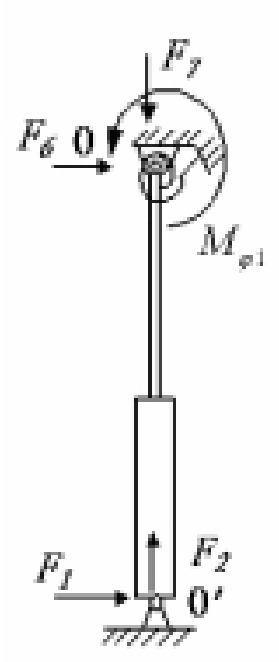

(a) in the wheel plane

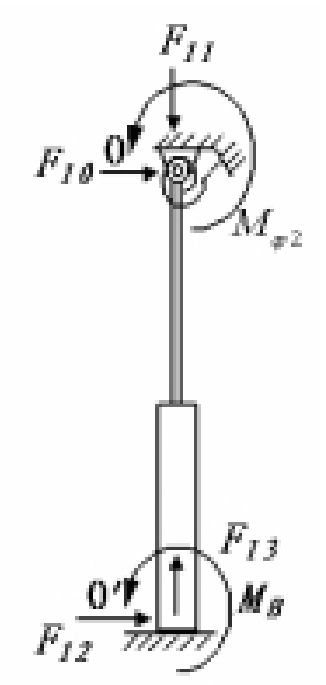

(b) in the axis plane
Fig. 4. (a) in the wheel plane (b) in the axis plane Equilibrium of forces on the rear absorber.

Since the piston rod surface is subjected to a dynamic friction and suffers serious wear-out in the lateral direction of the car, the absorber begins to leak damping oil. Due to some types of surface damage of the piston rod, consequently, a large economic loss has to be encountered by both the car maker and users. It is therefore necessary to model the suspension in association with the twin-tube hydraulic shock absorber. For 


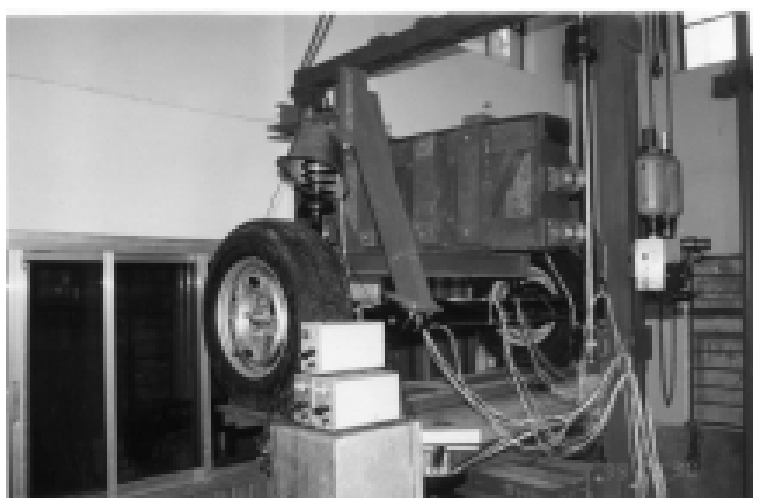

Fig. 5. The experimental set-up for bench tests of the rear dead axle, suspensions and absorbers.

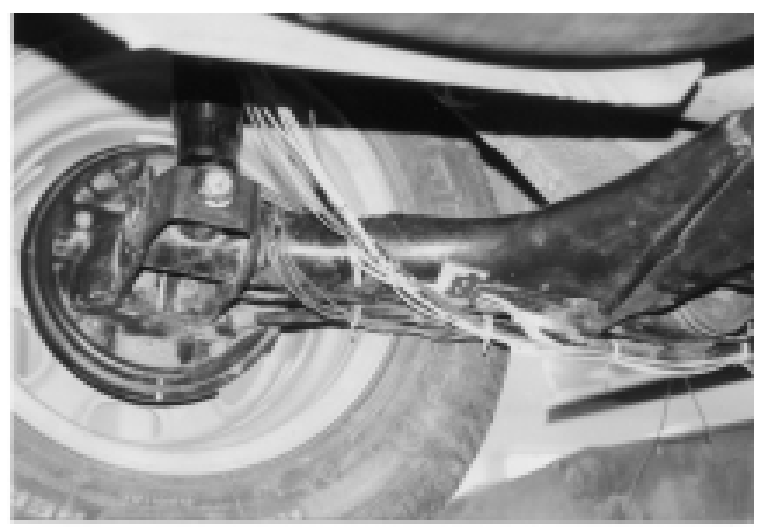

Fig. 6. Acceleration sensors and strain gauges for real road tests of the rear absorber and suspension on the left of the car.

a total evaluation of the lateral friction conditions and dynamic forces, hundreds of the rear shock absorbers that were replaced of surface damage from real applications and road tests are examined. Accelerations and strains of the piston rod in both the wheel and axle planes are measured through a number of bench tests of the brand new absorbers. The experimental set-up for bench tests of lateral and vertical accelerations and stresses of the rear shock absorber in the suspensions is shown in Fig. 5 and the real road test with acceleration sensors and strain gauges for the rear trailing arm, suspension and absorber on the left side of the car is shown in Fig. 6. Real time responses are plotted, respectively, in Fig. 7 for vertical accelerations of the shock absorber in the test rig and in Fig. 8 for strain measures of the piston rod in the real road test. Two couples of strain gauges are placed symmetrically on the piston rod in the axle and wheel planes.

\section{Strength analysis of the piston rod under peak loading}

In the analysis, the basic hypotheses are made as follows [5]: (1) the tube does not deform in the motion course, (2) the piston rod undergoes small deflection subjected to linearly distributed bending stress, (3) original geometrical parameters of the piston rod are kept unchangeable during certain deformations, (4) the shock absorber vibration is the wheel type and (5) in comparison with the body, the mass of the piston rod is small, so that it is ignored in the dynamic analysis of the suspension.

\subsection{Analysis of forces in the wheel plane}

If the tube is subjected to a system of forces in equilibrium, as depicted in Fig. 9 (a), the equations of equilibrium of the tube may be written as follows

$$
\begin{aligned}
& I_{1} \varepsilon_{1}=F_{3} l_{4}+F_{1} l_{3}-F_{4} l_{2} \\
& m l_{5} \varepsilon_{1}=F_{1}+F_{3}-F_{4} \\
& l_{1} \varepsilon_{1}=a_{f a}
\end{aligned}
$$

where $F_{3}$ and $F_{4}$ are reaction forces acting on the top and middle of the tube by the piston rod, $F_{5}$ is the reaction force acting on the bottom of the tube by the damping resistance, $O_{c}$ denotes mass centroid of the tube, $\varepsilon_{1}$ is supposed to be rotational acceleration of the tube around Point $O^{\prime}, I_{1}$ is inertia moment of the tube with respect to Point $O, m$ is mass of the tube including oil, $a_{f a}$ is acceleration of the tube measured at Point $A$, and $l_{1}, l_{2}, l_{3}, l_{4}$ and $l_{5}$ are, respectively, geometrical dimensions given values in Table 1 while the piston rod moves up to the maximum extent.

If the piston rod, on the other hand, is subjected to a system of forces in equilibrium, as depicted in Fig. 9 (b), the equations of equilibrium of the piston rod can be presented in the following

$$
M_{\varphi 1}+F_{3} l_{4}=F_{4} l_{2}
$$

The solution of the associated Eqs (1), (2), (3) and (4) results in expressions of reaction forces $F_{3}$ and $F_{4}$ of the following form as

$$
\begin{aligned}
F_{3}= & \left(I_{1} l_{2} \varepsilon_{1}-m l_{5} l_{3} l_{2} \varepsilon_{1}-M_{\varphi 1}\left(l_{3}-l_{2}\right)\right) / \\
& \left(\left(l_{4}-l_{3}\right) l_{2}+\left(l_{3}-l_{2}\right) l_{4}\right)
\end{aligned}
$$




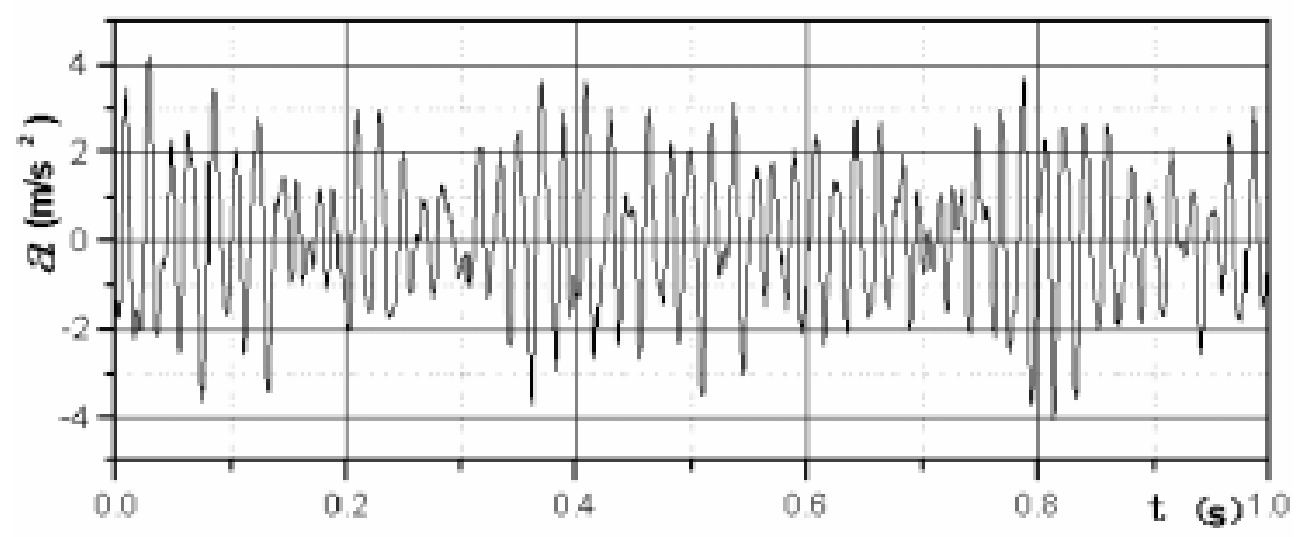

(a) In the wheel plane

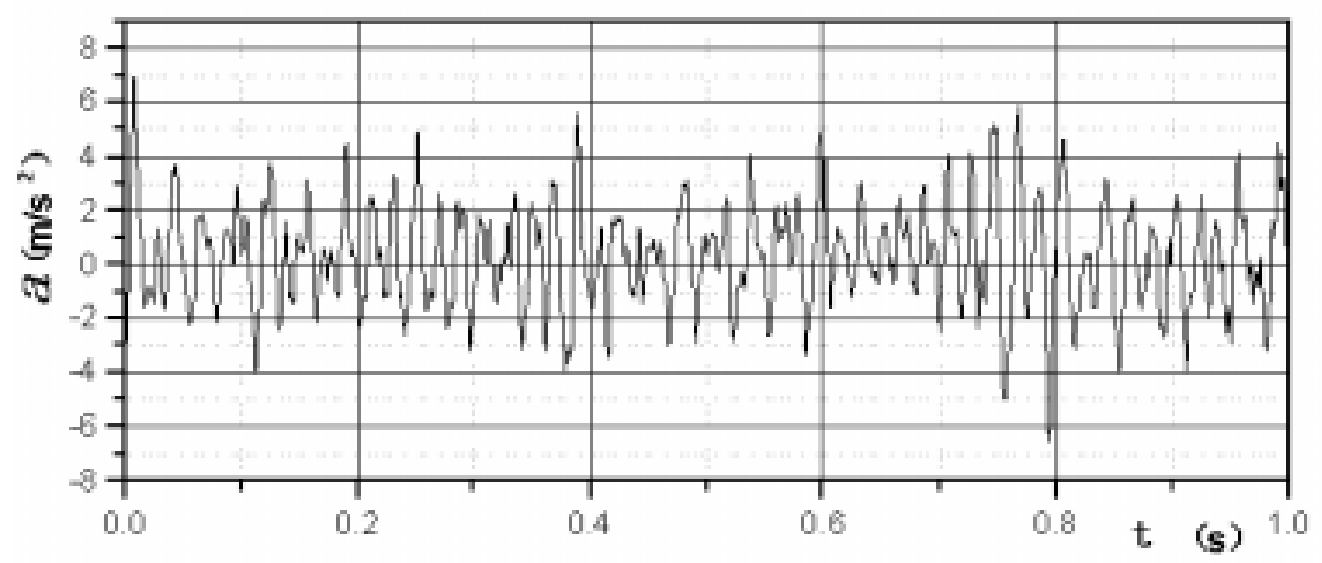

(b) In the axle plane

Fig. 7. (a) In the wheel plane (b) In the axle plane Real time responses of vertical accelerations for the tube of the rear absorber in the test rig.

$$
\begin{aligned}
F_{4}= & \left(I_{1} l_{4} \varepsilon_{1}-m l_{5} l_{4} l_{3} \varepsilon_{1}+\right. \\
& \left.M_{\text {varphi1 }}\left(l_{4}-l_{3}\right)\right) /\left(\left(l_{4}-l_{3}\right) l_{2}\right. \\
& \left.+\left(l_{3}-l_{2}\right) l_{4}\right) \\
\varepsilon_{1}= & a_{f a} / l_{1}
\end{aligned}
$$

By taking the maximum value from the test results as shown in Fig. 7(a), it is found that $F_{3}=159.5 \mathrm{~N}$ and $F_{4}=202.4 \mathrm{~N}$ according to Eqs (5), (6) and (7). The distribution of the moments acting on the piston rod in the wheel plane can be determined and are presented in Fig. 9(c).

\subsection{Analysis of forces in the axle plane}

If the tube is subjected to a system of forces in equilibrium, as depicted in Fig. 10 (a), the equations of equilibrium of the tube may be written as follows

$$
\begin{aligned}
& I_{2} \varepsilon_{2}=M_{B}+F_{9} l_{13}-F_{8} l_{11} \\
& l_{12} \varepsilon_{2}=a_{l b}
\end{aligned}
$$

where $F_{8}$ and $F_{9}$ are reaction forces acting on the top and middle of the tube by the piston rod, $F_{5}$ is the same as in Fig. 9, $\varepsilon_{2}$ is rotational acceleration of the tube around Point $O^{\prime}, I_{2}$ is inertia moment of the tube with respect to Point $O^{\prime}, a_{l b}$ is acceleration of the tube measured at Point $B$, and $l_{11}, l_{12}$ and $l_{13}$ are, respectively, geometrical dimensions given values in Table 1 whenever the piston rod moves up to the maximum extent.

If the piston rod, on the other hand, is subjected to a system of forces in equilibrium, as depicted in Fig. 10 (b), the equations of equilibrium of the piston rod can be presented in the following

$$
M_{\varphi 2}+F_{9} l_{2}=F_{8} l_{4}
$$




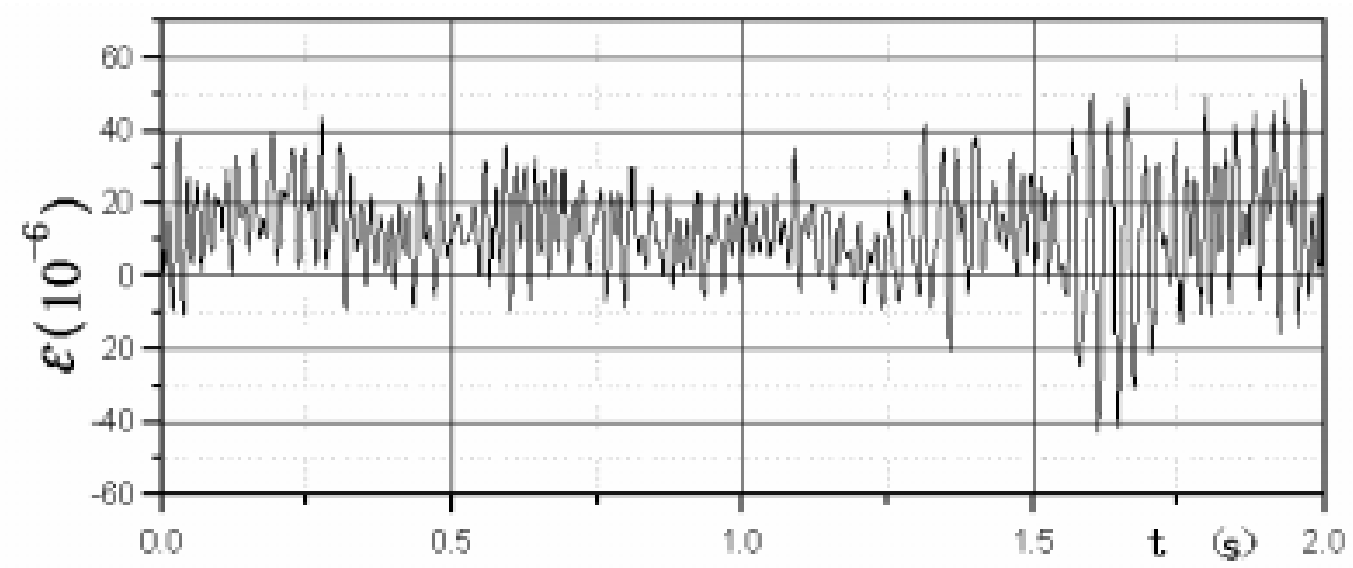

(a) In the wheel plane

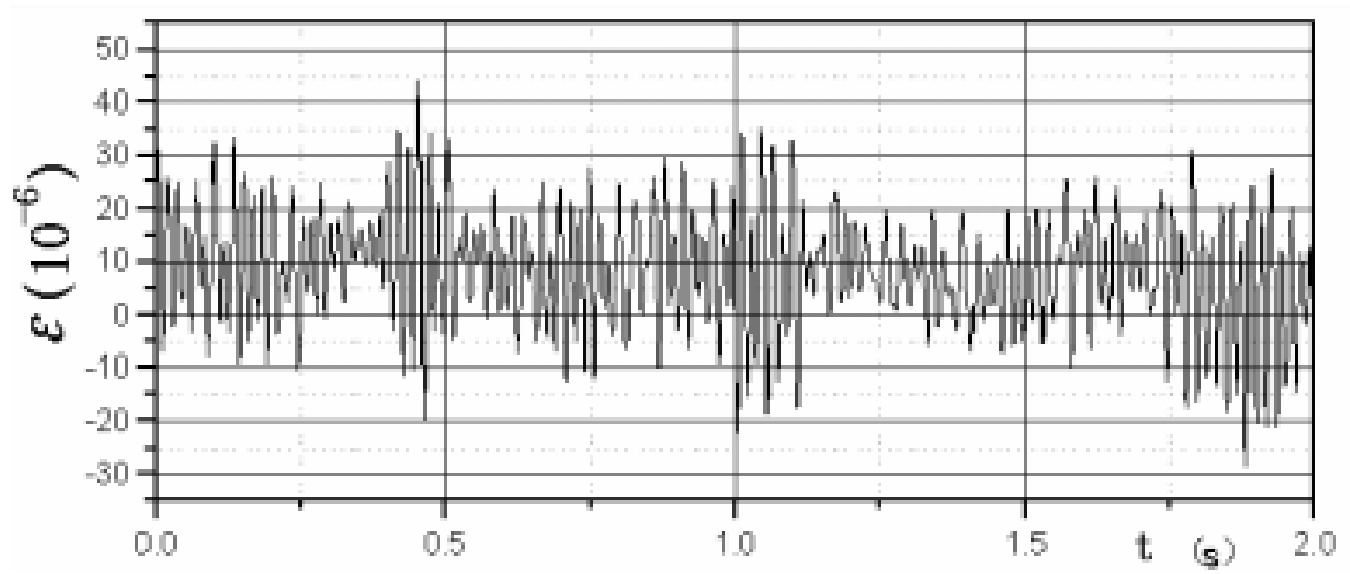

(b) In the axle plane

Fig. 8. (a) In the wheel plane (b) In the axle plane Real time responses of strains for the rear piston rod on uneven road at the speed of $80 \mathrm{~km} / \mathrm{h}$.

The solution of Eqs (8), (9) and (10) in homogeneous form results in reaction forces of the following form as

$$
\begin{aligned}
F_{8}= & \left(I_{2} l_{2} \varepsilon_{2}+M_{\varphi 2} l_{13}-M_{B} l_{2}\right) / \\
& \left(l_{4} l_{13}-l_{2} l_{11}\right) \\
F_{9}= & \left(I_{2} l_{4} \varepsilon_{2}+M_{\varphi 2} l_{11}-M_{B} l_{4}\right) / \\
& \left(l_{4} l_{13}-l_{2} l_{11}\right) \\
\varepsilon_{2}= & a_{l b} / l_{12}
\end{aligned}
$$

By taking the maximum test data $a_{l b}$ from the test results as shown in Fig. 7(b), it is found that $F_{8}=$ $277.1 \mathrm{~N}$ and $F_{9}=53.7 \mathrm{~N}$ according to Eqs (11), (12) and (13). The distribution of the moment acting on the piston rod in the axle plane can be determined and are shown in Fig. 10 (c). Geometrical and physical parameters used in the above analysis are presented in Table 1 for details.

It is supposed that the types of dynamic loadings applied to the piston rod in the wheel and axle planes are independent from each other. The diameter $d$ of the piston rod is known in Table 1, then the peak value of the normal stress to the cross section can be determined below

$$
\begin{aligned}
\sigma_{\max } & =\frac{M_{\max }}{W_{z}}=\frac{\sqrt{M_{\varphi 1}^{2}+M_{\varphi 2}^{2}}}{\pi d^{3} / 32} \\
& =368.7 \mathrm{MN} / \mathrm{m}^{2}<\sigma_{s}=450 \mathrm{MN} / \mathrm{m}^{2}
\end{aligned}
$$

where $W_{z}$ is bending stiffness of the cross section, $\sigma_{s}$ is the yield limit of material for the piston rod [5]. Therefore, the strength requirement for the piston rod is satisfied. 


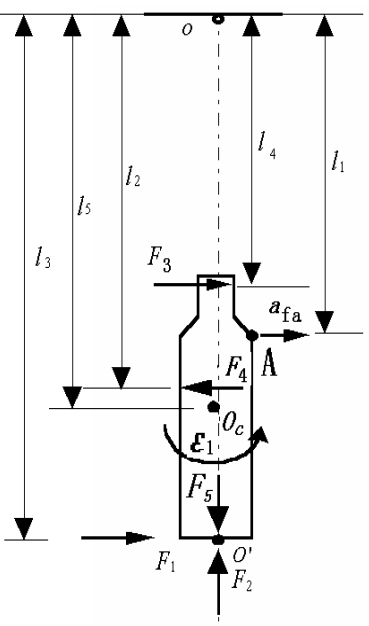

(a) The loads on the tube

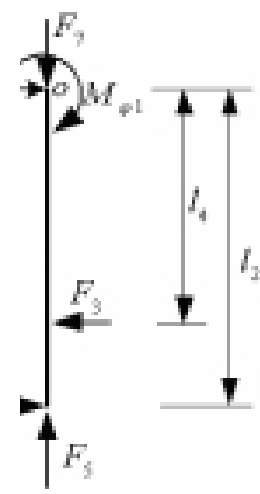

(b) The loads on the piston rod (c) Bending moments on the piston rod

Fig. 9. (a) The loads on the tube (b) The loads on the piston rod (c) Bending moments on the piston rod External and internal forces acting on the absorber in the wheel plane.

Table 1

Geometrical and physical parameters of the absorber

\begin{tabular}{ccll}
\hline Parameters $(\mathrm{mm})$ & Values & Parameters & Values \\
\hline$l_{1}$ & 420 & $\mathrm{~m}(\mathrm{~kg})$ & 2.237 \\
$l_{2}$ & 396 & $I_{1}\left(\mathrm{~kg} \cdot \mathrm{m}^{2}\right)$ & 0.0868 \\
$l_{3}$ & 682 & $I_{2}\left(\mathrm{~kg} \cdot \mathrm{m}^{2}\right)$ & 0.0725 \\
$l_{4}$ & 305 & $M_{\varphi_{1}}(\mathrm{~N} \cdot \mathrm{m})$ & 55 \\
$l_{5}$ & 502 & $M_{\varphi_{2}}(\mathrm{~N} \cdot \mathrm{m})$ & 167 \\
$l_{11}$ & 377 & $M_{B}(\mathrm{~N} \cdot \mathrm{m})$ & 95 \\
$l_{12}$ & 262 & $a_{\mathrm{fa}}\left(\mathrm{m} / \mathrm{s}^{2}\right)$ & 4.1 \\
$l_{13}$ & 286 & $a_{\mathrm{lb}}\left(\mathrm{m} / \mathrm{s}^{2}\right)$ & 7 \\
$d$ & 11 & & \\
\hline
\end{tabular}

\section{Strength analysis for the piston rod under circle loading}

Two types of loading conditions for the strength analysis of the piston rod are periodic and stochastic loadings. The load acting on the rear shock absorber on the test rig is sinusoidal, whereas the dynamic load acting on the piston rod is stochastic in road tests for the rear shock absorber. The strength analysis for the piston rod under random loading is performed in an average way, so the strength of the piston rod under the peak stress must be analyzed. Values of strength and material properties required for the piston rod in the circle loading are given in Table 2 for details.

\subsection{The fatigue strength of the piston rod under periodic loading}

The safety factor of the piston rod can be determined by means of values of the rod strength and material properties in Table 2 as

$$
n_{\sigma}=\sigma_{-1} /\left(\frac{K_{\sigma}}{\varepsilon_{\sigma} \beta} \sigma_{a}+\Psi_{\sigma} \sigma_{m}\right)=1.52>1
$$

It is obvious from Eq. (15) that the fatigue strength requirement for the piston rod is satisfied.

\subsection{The fatigue strength of the piston rod under stochastic loading}

According to strain measures for the rear piston rod on uneven road in Fig. 8, $\sigma_{i}$ and $n_{i}$ can be computed by statistically counting amplitudes of stresses [6] and are presented in Table 3 . The test times per year are easily determined to be total $3.6 \times 10^{6}$.

$$
\sum n_{i} \sigma_{i}^{n}=2.347 \times 10^{48}
$$

According to the statistics in Table 3, the safety factor for the fatigue strength of the piston rod can be expressed by means of Miner's theory of fatigue damage cumulation as follows

$$
\begin{aligned}
& \left(\sigma_{-1}\right)_{G} \frac{\varepsilon_{\sigma} \beta}{K_{\sigma}} \times \sigma_{-1}=276.21 \\
& n_{\sigma}\left(\sigma_{-1}\right)_{G} /\left(\frac{1}{N_{0}} \sum n_{i} \sigma_{i}^{n}\right)^{1 / n}=1.58>1
\end{aligned}
$$

From the above evaluation, the fatigue strength of the piston rod in the lateral direction satisfies the requirement of safety when the test road condition is good and the driving velocity is in a range between 30 and 


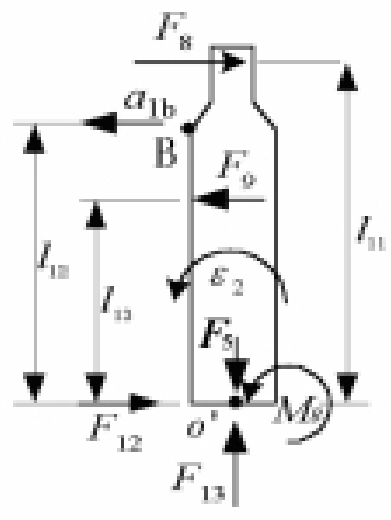

(a) The loads on the tube

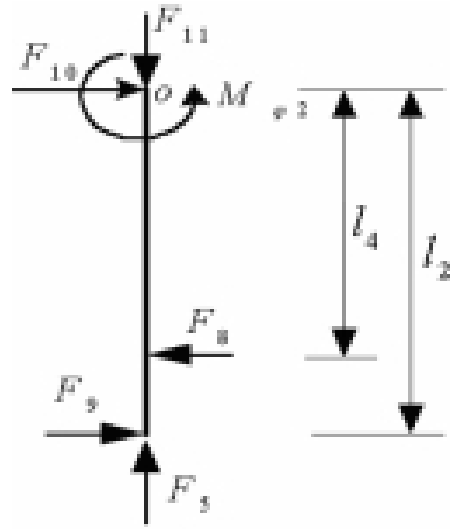

(b) The loads on the piston rod

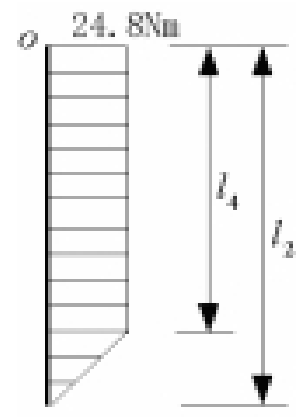

(c) Bending moments on the piston rod

Fig. 10. (a) The loads on the tube (b) The loads on the piston rod (c) Bending moments on the piston rod External and internal forces acting on the absorber in the axle plane.

Table 2

Values of strength and material properties for the piston rod

\begin{tabular}{lllc}
\hline Parameters $\left(\mathrm{MN} / \mathrm{m}^{2}\right)$ & Values & Parameters & Values \\
\hline Maximum alternating stress $\sigma_{\max }^{\prime}$ & 416 & Stress concentration coefficient $K_{\sigma}$ & 1.2 \\
Average alternating stress $\sigma_{\mathrm{m}}$ & 390.4 & Quality coefficient of the surface $\beta$ & 1.2 \\
Strength limit $\sigma_{b}$ & 650 & Sensitive coefficient of unsymmetrical circle $\Psi_{\sigma}$ & 0.43 \\
Permanent limit $\sigma_{-1}$ & 297 & Dimension coefficient $\varepsilon_{\sigma}$ & 0.93 \\
Amplitude of stress $\sigma_{a}$ & 25.6 & Number of loading circles for the fatigue $N_{0}$ & $10^{7}$ \\
Modulus of elasticity $E$ & $2.1 \times 10^{5}$ & Cotangent of the angle for fatigue $n$ & 18 \\
\hline
\end{tabular}

Table 3

The statistics of $\sigma_{i}$ and $n_{i}$ for fatigue strength of the piston rod in stochastic loading

\begin{tabular}{cccrr}
\hline Strain $\varepsilon\left(\times 10^{-6}\right)$ & Number of cycles per 2 seconds & Total strain $\varepsilon_{i}\left(\times 10^{-6}\right)$ & $\sigma_{i}=E \varepsilon_{i}\left(\mathrm{MN} / \mathrm{m}^{2}\right)$ & Number of cycles per yearn $n_{i}$ \\
\hline 5 & 25 & 769 & 161.5 & $9 \times 10^{7}$ \\
10 & 33 & 774 & 162.5 & $11.88^{7}$ \\
15 & 14 & 779 & 163.6 & $5.04 \times 10^{7}$ \\
20 & 7 & 784 & 164.7 & $1.52 \times 10^{7}$ \\
25 & 5 & 789 & 165.7 & $1.8 \times 10^{7}$ \\
30 & 2 & 794 & 166.8 & $0.72 \times 10^{7}$ \\
\hline
\end{tabular}

$$
\varepsilon_{\max }=\sqrt{\left(\varepsilon_{\text {dAxle }}+\varepsilon_{\text {smaxAxle }}\right)^{2}+\left(\varepsilon_{\text {dmaxWheel }}+\varepsilon_{\text {smaxWheel }}\right)^{2}}=2079 \times 10^{-6}
$$

$80 \mathrm{~km} / \mathrm{h}$. Further by numerical results computed for the fatigue strength of the piston rod under the periodic and statistical loads, the safety requirement of strength of the piston rod under consideration is satisfied and the safety margin for the strength is quite large.

\subsection{Strength analysis for the piston rod under peak strain}

The maximum strains of the piston rod subjected to random dynamic bending in real road test are computed by statistical procedure, based upon strain measures in
Fig. 8 and presented in Fig. 11 in which amplitudes of strains is increased by $5 \times 10^{-6}$. As shown in Fig. 11, the dynamic strain at the 14th is maximum which are considered both in the wheel plane and the axle plane. The static state strains can be obtained according to the average of measures three times in the real road test. Total strains are the dynamic strains plus corresponding the static strains. The strains are shown in the Table 4 for details.

The equivalent peak strain can be obtain as Eq. (19), where $\varepsilon_{\text {dmaxAxle }}$ and $\varepsilon_{\text {dmaxWheel }}$ are, respectively, dynamic strains in the axle and wheel planes, and $\varepsilon_{\text {smaxAxle }}$ and $\varepsilon_{\text {smaxWheel }}$ are, respectively, static 


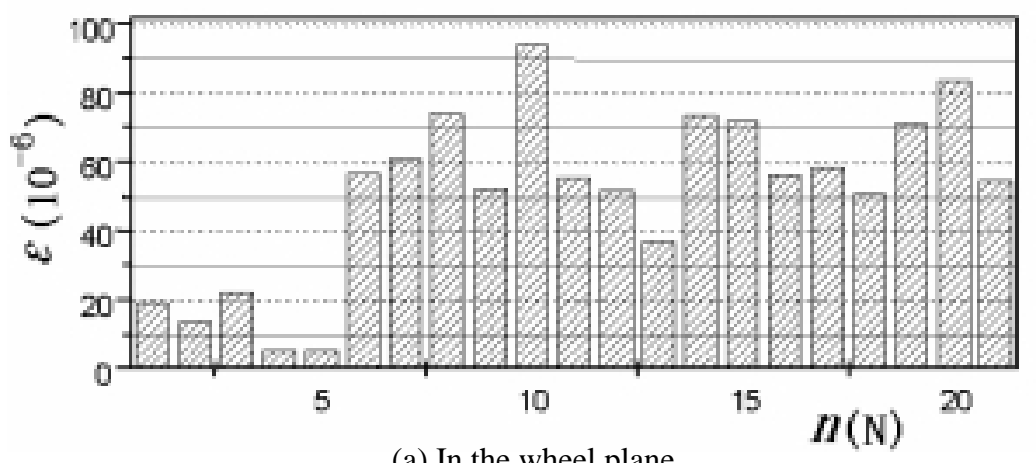

(a) In the wheel plane

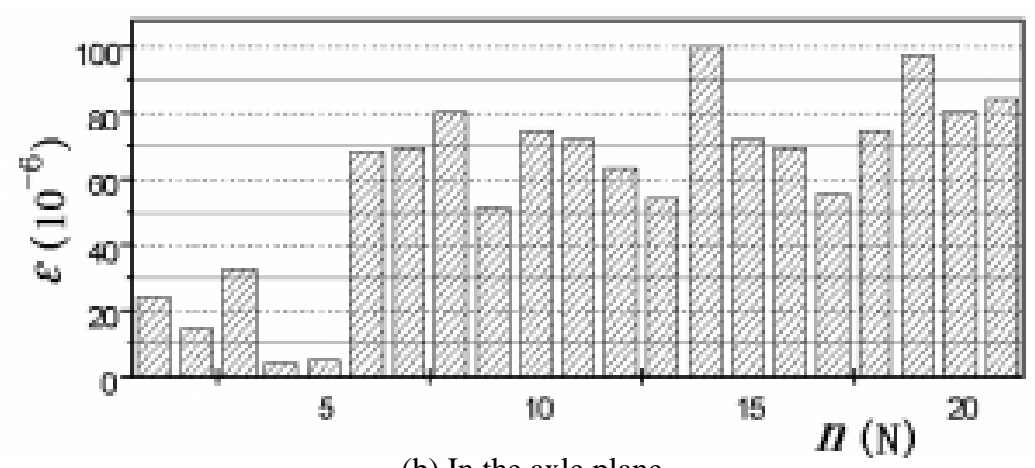

(b) In the axle plane

Fig. 11. (a) In the wheel plane (b) In the axle plane The statistics of the strain measures for the piston rod in real road test.

strains in the axle and wheel planes. Accordingly, the peak stress of the piston rod suffering the peak strain is simply obtained below

$$
\begin{aligned}
\sigma_{\max } & =E \times \varepsilon_{\max }=4366 \mathrm{MN} / \mathrm{m}^{2}<\sigma_{s} \\
& =450 \mathrm{MN} / \mathrm{m}^{2}
\end{aligned}
$$

It is indicated from Eq. (20) that the strength requirement for the piston rod subjected to the peak strain is satisfied. On the other hand, the peak stress in the stochastic loading is increased faster than that in the periodic loading and than that computed by the Miner's theory of fatigue damage cumulation. With increase of the car speed and riding toughness, the peak stress under stochastic load may increase further than that mentioned above, which may exceed the yield limit of the piston rod material and cause some problems.

From viewpoint of the whole car assembly, the rear shock absorbers may be hardly perfectly placed in their positions between the suspensions and body due to some manufacturing error and uncertainties. Consequently, the piston rods suffer initial bending because of lateral and vertical forces of car body and passengers. The car body vibrates up and down in the riding and additional dynamic loadings caused by the above
Table 4

The maximum strains of the piston rod

\begin{tabular}{lclc}
\hline $\begin{array}{l}\text { Strains in the } \\
\text { axle plane }\end{array}$ & $\begin{array}{c}\text { Vales } \\
\left(\times 10^{-6}\right)\end{array}$ & $\begin{array}{l}\text { Strains in the } \\
\text { wheel plane }\end{array}$ & $\begin{array}{c}\text { Values } \\
\left(\times 10^{-6}\right)\end{array}$ \\
\hline$\varepsilon_{\text {dmaxAxle }}$ & 100 & $\varepsilon_{\text {dmaxWheel }}$ & 73 \\
$\varepsilon_{\text {smaxAxle }}$ & 1475 & $\varepsilon_{\text {smaxWheel }}$ & 1284 \\
\hline
\end{tabular}

mentioned reasons are applied on the piston rod in the longitudinal and lateral directions. All these possibilities may easily produce the peak stress of the piston rod, which may not lead to a plastic failure of material, but cause intensive lateral frictions and surface wear-out of the piston rod.

\section{Conclusions}

Experiments including the rear suspensions bench test and the real car road test are made for acquirement of strain measures and acceleration signals of the twintube hydraulic shock absorber. Analysis of dynamic loads acting on the piston rod in both the wheel and axle planes is carried out by modeling the car rear suspension mechanically. According to the experimental data and real parameters, the fatigue strength of the piston 
rod is studied by consideration of two kinds of forces: the peak and circlic loadings.

By the results obtained from the real road tests and statistic solution for the shock absorber of the car riding on uneven road at the speed of $80 \mathrm{~km} / \mathrm{h}$, it is evident that the piston rod peak stresses increase dramatically and are related to velocities of the driving car and road conditions. If the assembly of the absorber components with the car body and suspension is not so accurate in position as it should be, the level of normal stresses may exceed the yield limit of material for the piston rod.

Because the absorber is a complex system itself from the viewpoint of vehicle systems dynamics, theoretical analysis is demanded in combination with necessary experiments to validate its design and application in real situations. In the present research, the systematic synthesis for the piston rod and seal of the absorber gives an insight into the friction induced failures and tribological design problems of the absorber.

\section{Acknowledgment}

The financial support to this work in parts by the Shanghai Administration of Education under Shanghai
Key Disciplines Development Fund Project and Shanghai Automotive Technology Development Foundation under Contract No.1325 A as well as the assistance for carrying out bench and road tests by National Key Laboratory for Vibration, Shock and Noise, Shanghai Jiao Tong University are greatly appreciated.

\section{References}

[1] Defu Yu and Zhao Fu, The outer-performance distortion of the suspension shock absorber and its critical velocity, Journal of Beijing Institute of Technology 16(4) (1995), 198-206.

[2] J.J. Harrigan, S.R. Reid and C. Peng, Inertia effects in impact energy absorbing materials and structures, Int. J. Impact Engineering 22(4) (1999), 955-979.

[3] Zhijun Cai, Dynamic simulation and experiment of rear suspension systems for SANTANA series cars, Master's Dissertation, Shanghai Jiaotong University, Shanghai, P. R. China, 2000

[4] C. Kim, P.I. Ro and H. Kim, Effect of the suspension structure on equivalent suspension parameters, IMechE J. Automobile Engineering 213(D4) (1999), 457-470.

[5] E.J. Hearn, Mechanics of material, Pergamon Press Ltd., Berlin, 1977.

[6] E.W. Roger, M.P. Miner, M.G. Michael et al, Measuring computer software reliability, Computer \& Industrial Engineering 2(3) (1978), 141-151. 

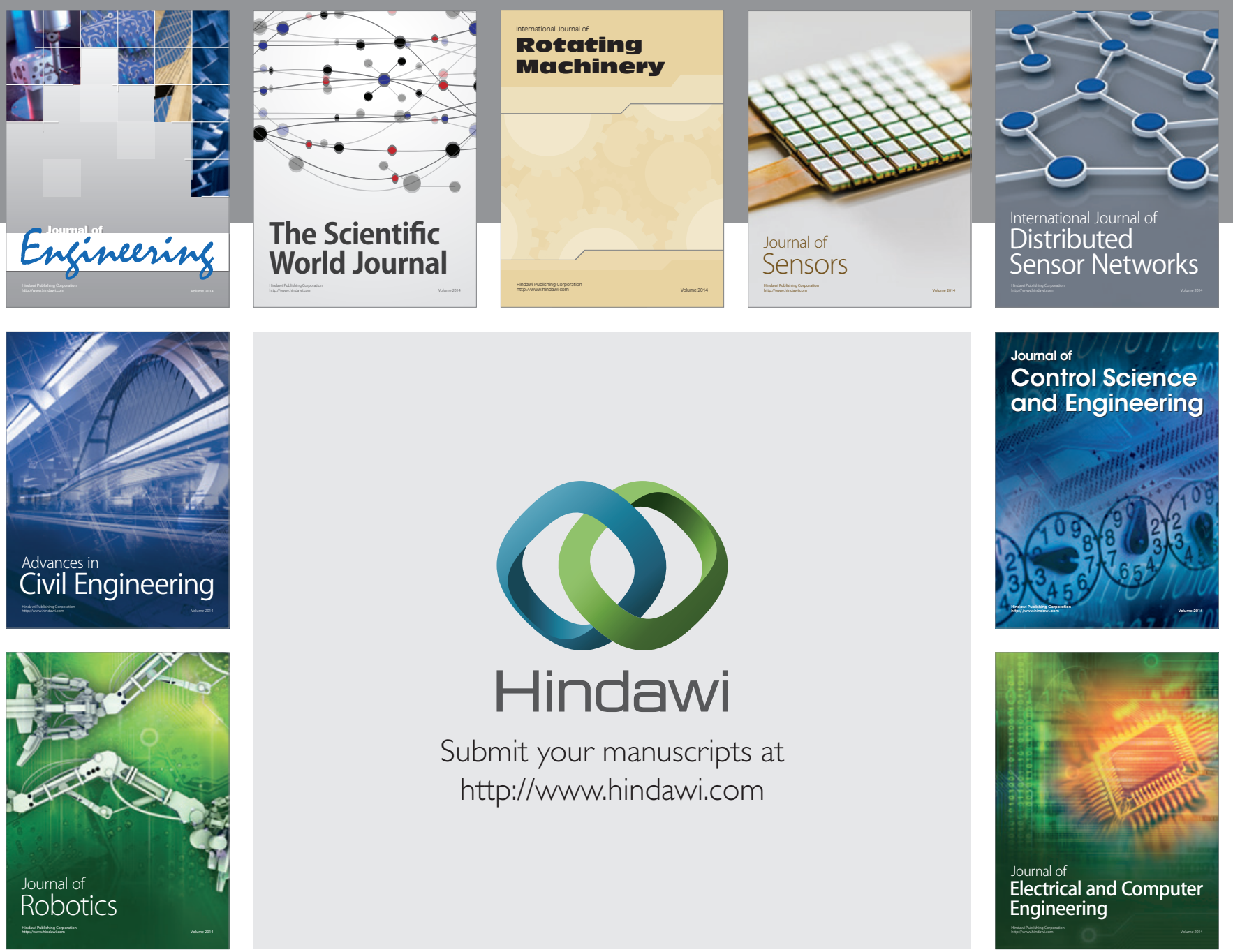

Submit your manuscripts at

http://www.hindawi.com
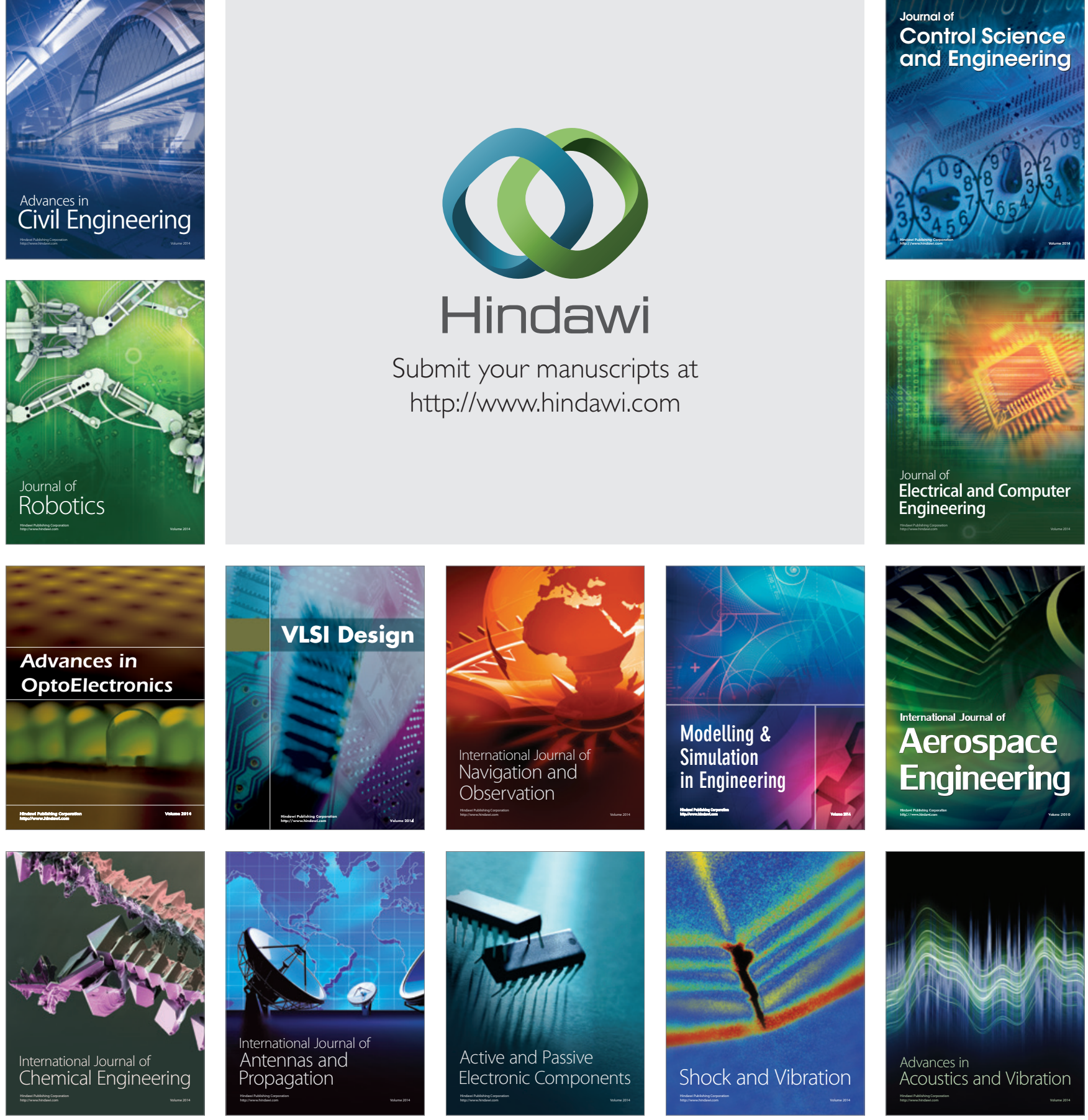